\title{
Tumor-agnostic precision oncology: striving to prioritize internationalization for the management of cancer patients
}

\author{
Yuko Kitagawa ${ }^{1,2}$
}

Published online: 5 May 2020

(c) Japan Society of Clinical Oncology 2020

The last 3 years have seen a paradigm shift in the regulatory approval of cancer treatments. With the approval of the first three agents, pembrolizumab and larotrectinib/entrectinib, for the treatment of solid tumors based on the presence of specific biomarkers rather than on tumor site, the precedent of tumor-agnostic precision oncology has been established. However, these approvals pose several clinical questions regarding the optimal use of tumor-agnostic agents. Uncertainties include the appropriate patient selection, timing, and methodology of microsatellite instability (MSI)/deficient mismatch repair (dMMR)/neurotrophic tyrosine receptor kinase (NTRK) testing, and also the sequence of administration of these agents in the treatment context of patients with MSI/dMMR or NTRK fusion-positive solid tumors. In consideration of the knowledge that the prevalence of MSI/ $\mathrm{dMMR}$ is low in most common solid tumors and the prevalence of NTRK fusions is extremely low, it is critical that clinicians understand whether all cancer patients should be tested, and if so, when, and using which test(s).

The recently published special article 'JSCO/ESMO/ ASCO/JSMO/TOS: International expert consensus recommendations for tumor-agnostic treatments in patients with solid tumors with microsatellite instability or NTRK fusions' [1], provided us with the opportunity, as the President of the Japan Society of Clinical Oncology (JSCO) at that time, to be able to supply answers to these key questions. This was the first worldwide consensus article focusing on tumoragnostic treatments in patients with solid tumors, and the content was developed based on a meeting of international experts organized by the JSCO in cooperation with the European Society for Medical Oncology (ESMO). In addition

Yuko Kitagawa

kitagawa.a3@keio.jp

1 Department of Surgery, Keio University School of Medicine, 35 Shinanomachi, Shinjuku, Tokyo 160-8582, Japan

2 The Japan Society of Clinical Oncology (JSCO), Tokyo, Japan to JSCO and ESMO, experts from the Japanese Society of Medical Oncology (JSMO), the American Society of Clinical Oncology (ASCO), and the Taiwan Oncology Society (TOS) were assembled [1]. The purpose of the meeting was to develop international consensus recommendations on the use of tumor-agnostic treatments in patients with solid tumors and to generate a reference document for clinical practice, for pharmaceutical companies in the design of clinical trials, for ethics committees in the approval of clinical trial protocols, and for regulatory authorities in relation to drug approvals, with a particular emphasis on diagnostic testing and patient selection. To support our recommendations, we also investigated the prevalence of MSI and NTRK fusions in common adult and pediatric solid tumors [1]. Using data from the Foundation Medicine database (FMI, Cambridge, MA, USA), 217,086 DNA samples (the largest number worldwide) obtained from a range of different solid tumor types were profiled. Additional genomic profiling of tumors to identify other potentially targetable alterations (such as BRCAness, homologous recombination deficiency [HRD], and tumor mutational burden [TMB]-high) and gene alterations (e.g., ALK, FGFR, HER2, HER3, KRAS, RET, $R O S 1)$ which could be used in future tumor-agnostic treatment approaches, is ongoing.

However, there are some conflicting data which add additional complexity to treatment decisions. For example, an outstanding response from BRAF inhibitor monotherapy in $B R A F$-mutant malignant melanoma has been reported [2], while, conversely, a poor response was seen in $B R A F$-mutant metastatic colorectal cancer [3]. Suboptimal response to BRAF inhibitor monotherapy has been linked to incomplete inhibition of mitogen-activated protein kinase (MAPK) signaling in colorectal cancer cell lines. BRAF inhibition results in rapid feedback activation of epidermal growth factor receptor (EGFR)-mediated MAPK signaling in in vitro studies of $B R A F \mathrm{~V} 600 \mathrm{E}$-mutant colorectal cancer cells, permitting sustained MAPK activation and continued cell proliferation [4]. Combinations of BRAF and EGFR 
inhibitors resulted in synergistic inhibition of tumor growth in BRAF V600E-mutant colorectal cancer xenograft models [4]; in the subsequent clinical study (BEACON), adequate activity of EGFR-targeted monoclonal antibodies combined with BRAF inhibition was established [5]. Thus, we have arrived at an era of focusing on the molecular biology of a tumor, and our approach to future drug development, whether tumor site-specific versus tumor-agnostic, must be altered accordingly.

Finally, as the past President of the JSCO, I want to express my sincere thanks to all the experts and the societies who participated in the generation of the consensus document, as well as to the FMI. The JSCO, a leading Japanese professional organization founded in 1963, has long been committed to advancing the specialty of clinical oncology and promoting a multidisciplinary approach in cancer treatment and care. More than 17,000 oncology practitioners currently belong to the JSCO. The number of members is continuing to increase and the JSCO is now the largest clinical oncology organization in Japan. The specialties of JSCO members encompass more than 20 diverse fields, and include surgeons, medical oncologists, urologists, gynecologists, radiologists and others. Thus, whilst praiseworthy, the JSCO acknowledges that this project is only the first step in creating a global consensus on cancer management, and we must continue to strive, as a society, to prioritize internationalization with other major societies and disciplines.

Funding None.

\section{Compliance with ethical standards}

Conflict of interest YK: fees for consultancy/advisory roles from ONO PHARMACEUTICAL CO., LTD., AsahiKASEI Co., Ltd., and Bristol-Myers Squibb; research funding from TAIHO PHARMACEUTICAL CO., LTD., CHUGAI PHARMACEUTICAL CO., LTD., Yakult
Honsha Co. Ltd., DAIICHI SANKYO COMPANY, LIMITED, Merck Serono Co., Ltd., AsahiKASEI Co., Ltd., EA Pharma Co., Ltd., Otsuka Pharmaceutical Co., Ltd., Takeda Pharmaceutical Co., Ltd., Otsuka Pharmaceutical Factory Inc., SHIONOGI \& CO., LTD., KAKEN PHARMACEUTICAL CO.,LTD., Kowa Pharmaceutical Co., Ltd., Astellas Pharma Inc., MEDICON INC., SUMITOMO DAINIPPON PHARMA Co., Ltd., Taisho Toyama Pharmaceutical Co., Ltd., Kyouwa Hakkou Kirin Co., Ltd., Pfizer Japan Inc., ONO PHARMACEUTICAL CO., LTD., NIHON PHARMACEUTICAL CO., LTD., Japan Blood Products Organization, Medtronic Japan Co., Ltd., and Sanofi K.K.; and grants from Eisai Co., Ltd., TSUMURA \& CO., KCI Licensing, Inc., ABBOTT JAPAN CO., LTD., and FUJIFILM Toyama Chemical Co., Ltd.

\section{References}

1. Yoshino T, Pentheroudakis G, Mishima S et al (2020) JSCO/ ESMO/ASCO/JSMO/TOS: International expert consensus recommendations for tumour-agnostic treatments in patients with solid tumours with microsatellite instability or NTRK fusions. Ann Oncol. https://doi.org/10.1016/j.annonc.2020.03.299Epub ahead of print

2. Chapman PB, Hauschild A, Robert $C$ et al (2011) Improved survival with vemurafenib in melanoma with BRAF V600E mutation. N Engl J Med 364:2507-2516. https://doi.org/10.1056/ NEJMoa1 103782

3. Kopetz S, Desai J, Chan E et al (2015) Phase II pilot study of vemurafenib in patients with metastatic BRAF-mutated colorectal cancer. J Clin Oncol 33:4032-4038. https://doi.org/10.1200/ JCO.2015.63.2497

4. Corcoran RB, Ebi H, Turke AB et al (2012) EGFR-mediated reactivation of MAPK signaling contributes to insensitivity of BRAFmutant colorectal cancers to RAF inhibition with vemurafenib. Cancer Discov 2:227-235. https://doi.org/10.1158/2159-8290. CD-11-0341

5. Kopetz S, Grothey A, Yaeger R et al (2019) Encorafenib, binimetinib, and cetuximab in BRAF V600E-mutated colorectal cancer. N Engl J Med 381:1632-1643. https://doi.org/10.1056/ NEJMoa1908075

Publisher's Note Springer Nature remains neutral with regard to jurisdictional claims in published maps and institutional affiliations. 